\title{
Tecnologías de Información y comunicación desde la virtualidad para la formación en investigación aplicada e innovación "caso semilleros de investigación en los programas tecnológicos Universidad de Caldas”
}

\section{Information and communication technologies from the virtuality for the training in applied research and innovation "case of research seeds in the technological programs Universidad de Caldas"}

\author{
Rubén Darío Cárdenas Espinosa ${ }^{1}$ \\ Universidad de Caldas, Colombia
}

Recibido: 20-02-2018

Aceptado: 22-06-2018

\section{Cita Recomendada}

Cárdenas, R. (2018). Tecnologías de Información y comunicación desde la virtualidad para la formación en investigación aplicada e innovación "caso semilleros de investigación en los programas tecnológicos Universidad de Caldas”. Hamut'ay, 5 (1), 105-117.

http://dx.doi.org/10.21503/hamu.v5i1.1562

\section{RESUMEN}

El propósito de este artículo fue presentar el uso de las tecnologías de información y comunicación desde la virtualidad en los programas Tecnológicos a Distancia de la Universidad de Caldas (República de Colombia) en los cuales se proporcionaron a los estudiantes los fundamentos teóricos y prácticos que les permitan el desarrollo de productos electrónicos aplicables que fomenten la conformación e inscripción en los semilleros de investigación de dichos programas. Se partió de que los estudiantes de las tecnologías a distancia carecen de competencias en investigación aplicada e innovación para conformar semilleros de investigación. Esta situación ocasiona que, los estudiantes no posean las habilidades para formular y ejecutar proyectos de emprendimiento e innovación que les brinde herramientas para enfrentar el entorno laboral. Por tal motivo, se aplicó una intervención basada en el Modelo PACIE (Presencia, Alcance, Capacitación, Interacción, E-Learning) bajo el supuesto de que desde la virtualidad sería posible realizar la conformación de semilleros de investigación y establecer, de esta manera, las competencias necesarias para que al graduarse puedan desarrollar proyectos de investigación aplicada e innovación que transformen su entorno laboral y el de sus comunidades. El tipo de investigación es cuantitativo, con diseño cuasiexperimental, de enfoque empírico - analítico, descriptivo; de corte transversal.

Como resultado se obtuvo que el grupo experimental después de la aplicación de la experimentación se situó en el nivel de logro destacado un $40 \%$ y el 30\% en logro previsto, a diferencia del grupo de control que solo el 60\% estuvo en proceso; así como también se implementó el Semillero de Investigación e Innovación TECSIS y RELEC y se desarrolló recursos desde las tecnologías de información

1 DSC. Ingeniería Electrónica, PhD. Tecnología de Información, Candidato a Doctor en Proyectos, Investigador Senior. Escritor, Mentor INNPULSA, Director Grupo de Investigación ReNuevaTe Ciencia, Tecnología e Innovación. Instructor SENA Distrito Capital Centro Metalemecánico. Catedrático Tutor de la Universidad de Caldas, Colombia; rdcardenas@gmail.com 
y comunicación articulados con herramientas como Blackboard Collaborate, Whatsapp, Neolms, Moodle, Blogger, Soundcloud, Youtube y Vimeo.

Palabras Clave: TIC, Entornos virtuales de aprendizaje, Innovación Tecnológica, Investigación Aplicada, Modelo PACIE.

\section{Abstract}

The purpose of this article is to present the use of Information and Communication Technologies from the virtuality in the distance technological programs of the University of Caldas (Republic of Colombia) in which the students were provided with the theoretical and practical foundations that allow the development of applicable electronic products, which would boost the formation and enrollment in research seedbeds within those programs. It was based on the fact that students of distance technologies lack of competences in applied research and innovation to develop research seedbeds. This situation restricts students to have the skills to formulate and execute entrepreneurship and innovation projects that provide them with tools to face the work environment.

For this reason, an intervention based on the PACIE Model (Presence, Scope, Training, Interaction, E-Learning) was applied, under the assumption that from the virtuality, it would be possible to develop research seedbeds and establish, in this way, the necessary skills so that upon graduation students can develop applied research and innovation projects that transform their work environment and that of their communities. The type of research is quantitative, with a quasi - experimental design, with an empirical - analytical approach; a descriptive cross-sectional study.

As a result, we observed that after the application of the experiment, $40 \%$ achieved the outstanding level, and $30 \%$ got the expected achievement level in the experimental group; unlike the control group in which only 60\% achieved the in-process level. Likewise, the Research and Innovation Seedbed TECSIS and RELEC was implemented. Besides, resources were developed from the information and communication technologies articulated with tools such as Blackboard Collaborate, WhatsApp, Neolms, Moodle, Blogger, Soundcloud, YouTube and Vimeo.

Keywords: ICT, Virtual Learning Environments, Technological Innovation, Applied Research, PACIE model.

\section{INTRODUCCIÓN}

La investigación, el desarrollo tecnológico y la innovación contribuyen a la mejora continua y desarrollo de todos los sectores económicos de un país (Salas-Arbeláez, Solarte \& Vargas, 2017). En el sector empresarial, especialmente cuando está organizado en cadenas productivas, redes, clústers u otro tipo de esquemas de cooperación, son ambientes propicios para la innovación por su capacidad de interrelacionar factores como conocimientos científicos y tecnológicos en favor de clientes, usuarios y mercados (Riaño, 2017).
Gracias al desarrollo que ha tenido la tecnología y la reducción en los costos de producción y de sus componentes, se ha logrado que el diseño de productos electrónicos sea accesible y facilite el desarrollo de múltiples prototipos y aplicaciones en diversos campos del conocimiento en áreas como la agricultura, la automatización industrial, el hogar, transporte y entretenimiento entre otros, así como su articulación en lo conocido como el internet de las cosas que permiten la integración de sensores, actuadores, dispositivos de control con los dispositivos móviles desde la internet (Carrasco, 2017). En este contexto, se hace necesario 
proporcionar a los futuros tecnólogos de los programas a distancia de la Universidad de Caldas (República de Colombia) herramientas metodológicas y técnicas de investigación, así como los ambientes dirigidos a la formación del talento humano que impacten en los procesos de investigación aplicada, desarrollo tecnológico e innovación.

A pesar de tener a nuestro alcance un bagaje de herramientas tecnológicas y las tecnologías de la información y comunicación los estudiantes de las áreas de especialidad de los programas tecnológicos de la Universidad de Caldas, desde su inicio hace más de 15 años, nunca han participado en la iniciativa de creación de semilleros de investigación, debido a no sentirse incluidos por sus dificultades en tiempo, espacio y recursos. Y considerando que la calidad educativa que se brinda a nivel universitario debe ser igual para todos aquellos que estén interesados en obtener un título profesional sin distinción de la modalidad de estudio que ellos elijan, sea presencial, a distancia o virtual, por lo que tomando en cuenta estos aspectos, en este estudio se propuso como objetivo general articular las tecnologías de información y comunicación (TIC) desde la virtualidad en los programas tecnológicos a distancia de la Universidad de Caldas (República de Colombia) incorporando en dichos programas los fundamentos teóricos y prácticos que les permita a los estudiantes el desarrollo de productos electrónicos aplicables y que fomenten la conformación e inscripción en los semilleros de investigación en las áreas de tecnologías. Y como objetivos específicos: i. Analizar los programas tecnológicos de la Universidad de Caldas, la selección de los grupos de estudiantes de los municipios de Manizales, Riosucio y La Dorada, los horarios de las actividades en campo y el espacio en el Ambiente Virtual de Aprendizaje para realizar la intervención metodológica competencias en investigación aplicada e innovación. ii. Establecer los niveles de aprendizaje de las competencias en investigación aplicada e innovación, de forma general para recolectar la información mediante la administración de una pre prueba en cuatro secciones y la aplicación del programa solo al grupo experimental, mediante las 32 sesiones de aprendizaje en las que se incluyó el uso de las herramientas tecnológicas. iii.
Diseñar técnicas didácticas activas que estimulen el pensamiento para la resolución de problemas simulados y reales utilizando el B - Learning y Modelo PACIE (Presencia, Alcance, Capacitación, Interacción, E-Learning), para el logro de aprendizaje en las competencias en investigación aplicada e innovación a través del ambiente virtual de aprendizaje que facilite la Universidad de Caldas. iv. Analizar la información recolectada al concluir la aplicación del programa a las cuatro secciones, para conocer si el programa mejoró el logro de aprendizaje en las competencias en investigación aplicada e innovación para los estudiantes. v. Validar las estrategias metodológicas didácticas activas del proceso de formación aplicado utilizando B-Learning, el Modelo PACIE y los recursos web 2.0 que se articularon.

\section{Tecnologías de Información y Comunicación}

Para hacer referencia a las tecnologías de información y comunicaciones (TIC) se deben considerar las diversas acepciones que ésta tiene como un elemento que ha ido mutando y cambiando con el paso de los años, en donde se le han incluido una variedad de elementos que han hecho que ingrese de una manera casi obligatoria en todos los ámbitos, desde lo académico a lo empresarial. La definición engloba aspectos que van desde ser herramientas teórico conceptuales, soportes y canales que procesan, almacenan, sintetizan, recuperan y presentan información de la forma más variada, hasta la gestión de la información y el Internet, (Aprende en Línea, 8 de abril de 2015). Además, al ser tecnologías digitales que facilitan la comunicación e información, por su accesibilidad e interconexión a la información online, que incluye una diversidad de características como interactividad, instantaneidad, innovación, interconexión, digitalización e inmaterialidad, multimedia, hipermedia, y conectividad, (Grande, Cañón \& Cantón, 2016; Rivera, 2011). Desde otra vertiente Ruiz, Mendoza \& Ferrer (2014) refieren que las TIC son herramientas de gestión del conocimiento al mejorar el aprendizaje, hacerlo significativo al estudiante al facilitar el intercambio de información científica, porque permiten el acceso a diversos contenidos lingüísticos y culturales de manera sincrónica y asincrónica entre 
estudiante-docente, con abundante fuente de información; por lo que según Farroñay \& Ancaya (2016 p.35) estas herramientas permiten que el aula tradicional se convierta en un nuevo espacio con actividades innovadoras, al tener el potencial de transformar los procesos de enseñanza aprendizaje como recursos y medios didácticos al facilitar el acceso a bibliotecas virtuales, libros y artículos electrónicos, bases de datos, comunicaciones por correo electrónico, uso de redes sociales y blogs entre otros, (Izquierdo, Juárez \& Salas, 2017).

Tecnologías de Información y Comunicación como herramienta articuladora de virtualidad

La comunicación virtual ha desplazado a la comunicación tradicional, gracias al desarrollo de las herramientas de comunicación virtual aplicables en diversos contextos y sobre todo en el uso como estrategia de aprendizaje en el aula. Los cambios originados por la irrupción de la tecnología en la educación desde el nivel básico hasta el nivel superior han influenciado a que las instituciones educativas integren las tecnologías al proceso de aprendizaje con la finalidad de mejorarlo (Chávez, Del Toro \& López, 2017). Dentro de estas tecnologías según Chávez, Del Toro \& López (2017, p.48) "el blog constituye una herramienta de comunicación virtual usada con frecuencia en la educación", para Gálvez, 2010, citado en Larreal, 2015, "es una página web en la que se integra texto, imágenes, hipervínculos, vídeos y muchos otros objetos, permitiendo que los usuarios puedan hacer labor de autores o escritores, puesto que mediante esta herramienta es posible contener mensajes de una o varias personas respecto a un tema específico y quienes lean el blog pueden participar mediante sus aportaciones o cuestionamientos, promoviendo la reflexión individual y colectiva" (Larreal, p. 723).

Porras (2017) plantea que los Edublogs (blogs educativos y colaborativos) se han transformado en espacios de comunicación entre profesor- estudiante yentrelos mismos alumnosen los cuales se comparten reflexiones y se gestiona el conocimiento, facilitando la comunicación. Los Edublogs constituyen una de las herramientas más representativas de la web 2.0. Marín-Díaz \& Gómez-Parra (2015) refieren que la finalidad del edublog es fomentar el trabajo colaborativo y apoyar el aprendizaje de una materia de contenido.

Los recursos educativos digitales como los blogs articulados a las estrategias de enseñanza - aprendizaje desde los semilleros de investigación en las diversas plataformas de administración de aprendizaje como Neolms y Moodle constituyen una herramienta valiosa para complementar los diversos cursos que se orientan desde el campus virtual de la Universidad de Caldas.

Las redes sociales son otro tipo de mediaciones desde la virtualidad, al respecto Porras (2017) destaca el uso de redes sociales o comunidades como potenciadores de los procesos de enseńanza-aprendizaje al emplear recursos web 2.0 para establecer contactos, compartir información, jugar, ejecutar actividades de colaboración, chatear, compartir aficiones, comprar y vender productos. En el caso de WhatsApp y Blogger conforman un ciberespacio adecuado para comunicarse y en una estrategia que gestiona los procesos educativos del Siglo XXI al facilitar y permitir el aprendizaje colaborativo y el intercambio de información en comunidades de aprendizaje y cooperación.

Para Izquierdo, Juárez \& Salas (2017) es necesario realizar una reflexión sobre la forma en que el docente incentiva a sus estudiantes para emplear las TIC en todo su proceso investigativo y la forma de valerse de las mismas para generar una cultura digital con la apropiación de estos medios como software y las bases de datos especializadas en cada uno de los elementos del proceso de investigación con el fin de contribuir y enriquecer las acciones de colaboración entre grupos.

Estos medios brindan la experiencia a cada docente al integrar interactividad en sus prácticas educativas, desarrollar repositorios de trabajo para los estudiantes, compartir recursos didácticos para utilizarlos en clase para motivar e incentivar su ejercicio educativo, (Rojas, 2017).

Aprendizaje apoyado en Tecnologías de la Información y Comunicación

Para Morales \& Pereida (2017) el aprendizaje significativo requiere de estrategias didácticas cuyo centro es el aprendizaje del estudiante; éstas son 
herramientas pedagógicas que contribuyen a un aprendizaje profundo y permanente, propician el desarrollo del pensamiento crítico, establecen habilidades cognitivas y actitudes desde una perspectiva constructivista y significativa.

El Instituto Tecnológico y de Estudios Superiores de Monterrey (2010) señala que la técnica didáctica es un procedimiento que se presta para ayudar a realizar una parte del aprendizaje que se persigue con la estrategia. La estrategia abarca a la técnica y en el proceso de la técnica (instruccional o grupal), se contemplan las actividades necesarias para conseguir sus resultados. Dichas actividades son aún más parciales y específicas que la técnica y varían según el tipo de técnica o el tipo de actores con quien se desarrolla. Pueden ser aisladas y estar definidas por las necesidades de aprendizaje del grupo y es ahí donde el diseñador de contenidos apoyados en TIC requiere de la asesoría de un experto disciplinar; para considerar no sólo los tipos de técnicas didácticas, sino también las actividades que deberán solicitarse al estudiante, acordes con la competencia que se pretende alcanzar y sus estilos de aprendizaje, lo cual permite integrar estos aspectos en los semilleros de investigación.

\section{Semilleros de Investigación}

Este siglo XXI tan cambiante, ha obligado a que la academia, empresa e investigación formen una trilogía, para tener resultados palpables que permitan que los países puedan emergen. En cuanto a mejoras financieras se ha tornado imprescindible que se enfatice en la formación en investigación, por lo que una de esas estrategias son los semilleros de investigación, como lo aseveran Villalba \& Gonzáles, 2017; López \& Toro 2017, los semilleros son una nueva estrategia académica que permite al estudiante aprender a aprender, aprender a investigar y desarrollar conocimiento haciendo y que se formen futuros investigadores. Por lo que, según Llamas, 2015; UEB, 2013, los semilleros de investigación generan condiciones iniciales para la germinación de futuros investigadores para constituir profesionales que desde la investigación den respuesta a la complejidad de problemas de nuestra sociedad. No solo contribuye a los procesos de registro calificado y de alta calidad ante el Ministerio de Educación Nacional, sino que permite desarrollar competencias en investigación, innovación y emprendimiento, abriendo una oportunidad para realizar divulgación y producción científica e innovadora; ya que un semillero de investigación es una unidad de investigación y comunidad de aprendizaje en la que se reúnen estudiantes y docentes especialistas, con el propósito de desarrollar competencias investigativas dentro de la universidad, para la cual desarrollan y ejecutan proyectos de investigación sobre problemáticas específicas, (Alvites-Huamaní, 2015).

Para López \& Toro, 2017; Padilla, Rincón \& Buitrago, 2015, la investigación formativa se logra a través de los semilleros de investigación, porque desde aquí se pone en práctica la teoría a través de todas las acciones que favorecen verter los conocimientos, habilidades y actitudes al plantear soluciones viables a problemáticas actuales, regionales y del país.

Competencias en investigación aplicada e innovación

Aguerrondo (2017) hace referencia al término competencia, como el grupo de propiedades que cada individuo modifica de forma permanente y que requiere estar a prueba de la resolución de problemas concretos, tanto en la vida diaria como en el trabajo, la cual presenta incertidumbre y complejidad técnica. En el caso de los centros de educación, la competencia no proviene solamente de la aprobación de un plan de estudios, sino de la aplicación práctica de conocimientos necesarios para la resolución de problemas que no se pueden transmitir de manera mecánica; mezclan conocimientos tecnológicos previos y de la experiencia a partir de la práctica o del trabajo investigativo y académico. Unido a las competencias, esta la investigación, que según Lozada (2014) el propósito de la investigación es la generación de conocimiento que se puede emplear directamente y a mediano plazo en la sociedad o en el sector productivo. Esta se encarga del proceso de enlace entre la teoría y el producto, se desarrolla en 3 etapas para concebir productos viables en el comercio que puedan satisfacer las necesidades sociales 
previamente identificadas:

1. Inicio (búsqueda de aplicaciones y adaptación de teorías o resultados de las ciencias básicas);

2. Inclusión de necesidades sociales o industriales (permite inventar conceptos de aplicación de la teoría según las características del usuario final de la tecnología para asegurar la aceptabilidad y la usabilidad del concepto);

3. Proceso investigativo de maduración y transferencia de la tecnología (facilita la creación de prototipos que materializan el concepto y que se pueden transferir a la industria para que se transformen en productos).

Ramírez \& Vega (2015) plantean la innovación como la presentación o mejora de un nuevo producto, servicio, proceso, método de comercialización u organizativo, en una organización, lo cual complementa la investigación.

El manual de Oslo (De Oslo, 2006), clasifica la innovación en cuatro niveles: i. Primer Nivel. Innovación de producto (cambios en sus características); ii. Segundo Nivel. Innovación de proceso (cambios metodológicos en producción o distribución); iii. Tercer Nivel. Innovación organizacional (nuevas metodologías de organización); iv. Cuarto Nivel. Innovación de mercadotecnia (nuevas metodologías de comercialización).

Desde nuestra perspectiva las competencias en los estudiantes deben ser vinculantes de manera directa con la formación en investigación y la inclusión de innovación en todos los procesos educativos.

\section{Materiales Y Métodos}

\section{Participantes}

La muestra estuvo conformada por estudiantes de estrato social 1, 2 y 3, activos en las áreas de Tecnologías residentes de los municipios de Manizales, Riosucio y La Dorada del Departamento de Caldas en los Centros Regionales de Educación Superior CERES, se seleccionó una muestra intencionada del $25,33 \%$ de la población de estu- diantes inscritos en las asignaturas Electivas 1, 3 y Electrónica 2 (el muestreo fue no probabilístico, ver Tabla 1$)$.

Tabla 1. Descripción de la Muestra de Estudio

\begin{tabular}{lll}
\hline \multicolumn{1}{c}{ Grupos } & \multicolumn{1}{c}{ Secciones } & Cantidad \\
\hline Experimental & 5 Electiva 1 Dorada & 22 \\
\hline Control & 6 Electiva 3 Dorada & 16 \\
\hline Control & 6 Electiva 3 Manizales & 21 \\
\hline Experimental & 4 Electrónica 2 Riosucio & 17 \\
\hline & Total & 76 \\
\hline
\end{tabular}

Fuente: Registro de Universidad de Caldas (2017)

\section{Instrumento}

Los Instrumentos de recolección de datos utilizados en la investigación fueron dos. La Observación directa mediante Lista de Chequeo, los cuales cuentan con la validación del asesor de tesis doctoral del autor de la Universidad Internacional Iberoamericana de México (UNINI) y los pares académicos del grupo de Investigación ReNuevaTe Ciencia, Tecnología e Innovación. Y para ver las diferencias entre grupos se utilizó la prueba de "Desarrollo mis habilidades investigativas con TIC", descrita en la tabla 2.

La prueba permite conocer los niveles de logro de los estudiantes en el área de investigación aplicada, desarrollo Tecnológico e Innovación utilizando un recurso TIC, teniendo en cuenta los aspectos que requiere la formulación de un proyecto de investigación aplicada según RREDSI (Red Regional de Semilleros de Investigación) y de innovación, como se presenta en la tabla 3. 
Tabla 2. Prueba Desarrollo mis habilidades investigativas con TIC

\begin{tabular}{|c|c|}
\hline Dimensiones & Indicaciones \\
\hline $\begin{array}{l}\text { Evalúa una idea de proyec- } \\
\text { to de investigación aplica- } \\
\text { da, desarrollo Tecnológico } \\
\text { e Innovación utilizando un } \\
\text { recurso TIC }\end{array}$ & $\begin{array}{l}\text { 1. Evalúa su idea de proyecto respon- } \\
\text { diendo la pregunta ¿Qué voy a ha- } \\
\text { cer? } \\
\text { 2. Evalúa su idea de proyecto respon- } \\
\text { diendo la pregunta ¿Por qué lo voy } \\
\text { a hacer? } \\
\text { 3. Evalúa su idea de proyecto respon- } \\
\text { diendo la pregunta ¿Cuáles son sus } \\
\text { Beneficios? } \\
\text { 4. Evalúa su idea de proyecto respon- } \\
\text { diendo la pregunta ¿Qué necesida- } \\
\text { d(es) satisface? } \\
\text { 5. Evalúa su idea de proyecto respon- } \\
\text { diendo la pregunta ¿Quién pagaría } \\
\text { por éste y cómo se monetiza o finan- } \\
\text { cia? } \\
\text { 6. Evalúa su idea de proyecto res- } \\
\text { pondiendo la pregunta ¿Es factible } \\
\text { obtener un prototipo funcional en } 6 \\
\text { meses? } \\
\text { 7. Evalúa su idea de proyecto respon- } \\
\text { diendo la pregunta ¿Quién decide? } \\
\text { 8. Evalúa su idea de proyecto respon- } \\
\text { diendo la pregunta ¿Qué conozco } \\
\text { que sea similar? }\end{array}$ \\
\hline $\begin{array}{l}\text { Valida una idea de proyecto } \\
\text { de investigación aplicada, } \\
\text { desarrollo Tecnológico e } \\
\text { Innovación utilizando un } \\
\text { recurso TIC }\end{array}$ & $\begin{array}{l}\text { 1. Desarrolla una Ficha Bibliográfica } \\
\text { con mínimo } 5 \text { Referencias por es- } \\
\text { tudiante de búsquedas realizadas a } \\
\text { través de Google Académico https:/l } \\
\text { scholar.google.es/ como muestra el } \\
\text { siguiente cuadro } \\
\text { 2. Elabora un Modelo o Esquema de } \\
\text { Entrevista o Encuesta Elaborada (Li- } \\
\text { breto guía o Preguntas realizadas). } \\
\text { 3. Realiza un Mapa de empatía para } \\
\text { identificar elementos esenciales de } \\
\text { validación de la idea de proyecto } \\
\text { para contrastarla en campo a través } \\
\text { de una Encuesta o Entrevista. } \\
\text { 4. Presenta Resultados de Aplicación } \\
\text { Encuesta o Entrevista aplicada míni- } \\
\text { mo a } 5 \text { personas o empresas según } \\
\text { el tipo de idea } \\
\text { 5. Realiza la Validación Final de la } \\
\text { Idea: Respuesta a las siguientes } \\
\text { preguntas: a. "¿QUÉ SATISFACE? } \\
\text { (USUARIOS)"; b."¿QUIÉN PAGA- } \\
\text { RÍA? (CLIENTES)"; C." ¿HAY IDEAS } \\
\text { SIMILARES? (BENCHMARK)"; d."¿i }\end{array}$ \\
\hline $\begin{array}{l}\text { Presenta Escenarios de } \\
\text { Uso "Storyboards del } \\
\text { producto" para presentar } \\
\text { una idea de proyecto de } \\
\text { investigación aplicada, } \\
\text { desarrollo Tecnológico e } \\
\text { Innovación utilizando un } \\
\text { recurso TIC }\end{array}$ & $\begin{array}{l}\text { 1. Describe a través de imágenes y fra- } \\
\text { ses cortas el escenario de uso de su } \\
\text { idea de proyecto. } \\
\text { 2. Emplea recursos TIC para el desarro- } \\
\text { llo del Escenario de Uso de su idea } \\
\text { de proyecto. }\end{array}$ \\
\hline
\end{tabular}

Fuente: Elaboración propia (2017)
Tabla 3. Niveles de Evaluación de la prueba

\begin{tabular}{lll}
\hline Nivel & \multicolumn{1}{c}{ Criterios de Evaluación } & \multicolumn{1}{c}{ Calificación } \\
\hline 4 & Alto & Destacado \\
\hline 3 & Medio & Previsto \\
\hline 2 & Bajo & En proceso \\
\hline 1 & Nulo & Inicio \\
\hline
\end{tabular}

Fuente: Elaboración propia (2017)

\section{Tipo y diseńo}

El tipo de investigación es cuantitativo, porque analiza los resultados en porcentajes entre dos grupos antes y después de aplicar una nueva metodología, con diseño cuasiexperimental porque estuvo orientado a conocer y explicar las diferencias en porcentajes entre los grupos de control y experimental,

El enfoque es Empírico - Analítico y lo representa la elaboración de explicaciones a los fenómenos de la realidad que se buscan sean controlados y/o transformados por el hombre (Lazarsfeld, 1973). Y descriptivo al dirigirse fundamentalmente a la descripción de fenómenos sociales o educativos en una circunstancia temporal y espacial determinada. Los diferentes niveles de investigación varían en el tipo de pregunta que pueden formular (Cauas, 2015). La investigación es de corte transversal porque a la hora de la recolección de información se hizo de una sola vez, al haberse aplicado en un solo momento el instrumento de recolección de datos al inicio y al final del estudio e inmediatamente se procedió a su descripción y análisis. En la tabla 4 se puede observar el esquema del diseńo.

Tabla 4. Descripción de diseño de Estudio

\begin{tabular}{cccc}
\hline \multicolumn{4}{c}{ Diseño cuasiexperimental } \\
\hline M1 & 01 & $X$ & 02 \\
\hline M2 & 03 & - & 04 \\
\hline
\end{tabular}

Fuente: Elaboración propia (2017)

Donde:

M1: Grupo experimental

O1: Pre prueba

O2: Post prueba

$\mathrm{X}$ : Tratamiento o estímulo con la variable 
intervención metodológica competencias en investigación aplicada e innovación

M2: Grupo de control

O3: Pre prueba

O4: Post prueba

- : Ausencia de tratamiento o estímulo

Las variables de estudio son:

- Variable independiente (X): intervención metodológica competencias en investigación aplicada e innovación

- Variable dependiente (Y): Aprendizaje en el área de investigación aplicada, desarrollo Tecnológico e Innovación.

\section{Procedimiento}

Las estrategias didácticas apoyadas en TIC son los escenarios, procesos, actividades y experiencias desarrolladas junto a los estudiantes, para construir y reconstruir el conocimiento (Gualsaqui, 2015), ajustándolas para generar un proceso de inclusión tecnológica educativa que articule el B-Learning y el Modelo Presencia, Alcance, Capacitación, Interacción y E-Learning PACIE en programas tecnológicos de la Universidad de Caldas. Se realizó un plan de trabajo que se compone de tres fases de acompañamiento y asistencia permanente a todos los programas tecnológicos de los CREAD (Centro Regional de Educación a Distancia) de los Municipios y de la ciudad de Manizales.

\section{La Fase 1: Sensibilización y Motivación}

Se solicitó la participación de voluntarios de la comunidad académica: para el acompañamiento de los Semilleros de Investigación en la noche o fin de semana que los estudiantes no tengan formación.

Se organizó para el acompañamiento en campo: Se participó en las clases designadas por el Coordinador de Programa y el docente tutor en las asignaturas que requirieron acompańamiento metodológico para la formulación, estructuración, seguimiento, evaluación y documentación necesaria.

\section{La Fase 2: Consolidación y Producción}

Se consolido la creación de asignaturas en investi- gación aplicada e innovación: Se realiza la formulación de la asignatura con base al plan institucional de actividades académicas establecido por la Universidad de Caldas, en donde se contempla el número de créditos académicos, justificación, objetivos, contenidos temáticos, intensidad horaria, bibliografía, metodología, entre otros. Para lo que se solicitó a los coordinadores de los programas tecnológicos la selección de los grupos para realizar la investigación.

Se coordinó con el área de tecnología en electrónica los grupos de estudiantes de los municipios de Manizales, Riosucio y La Dorada, los horarios de las actividades en campo y el espacio en el ambiente virtual de aprendizaje para realizar la intervención metodológica en competencias en investigación aplicada e innovación durante el primer semestre de 2017.

\section{Fase 3: Proyección y resultados}

Se ejecutó el estudio entre los meses de febrero y abril de 2017. A partir de la entrevista con la Dirección de programa se estableció aplicar la investigación con los estudiantes de 4, 5 y 6 Semestre que se inscribieron en las asignaturas Electiva 1, Electiva 3 y Electrónica 2 de los Municipios de Manizales, Riosucio y La Dorada.

Según los lineamientos que tiene la Red Regional de Semilleros de Investigación RREDSI, al cual está vinculado la Universidad de Caldas y en la que nunca han participado los programas tecnológicos de la institución al carecer de semilleros, se establecieron las competencias en investigación aplicada e innovación permitiendo así la conformación del Semillero de Investigación de la tecnología en electrónica, para lo cual se programaron 32 sesiones para el grupo experimental aplicándoles las herramientas tecnológicas desde la virtualidad.

Se aplicaron las técnicas didácticas activas como lluvia de ideas, exposición, aprendizaje basado en problemas, estudio de caso, simulación, panel de discusión, método de proyectos, juego de roles, entrevistas, foro y simposio, los cuales se dinamizaron a través del Ambiente Virtual de Aprendizaje Moodle, la Red Social Whatsapp, Blackboard Collaborate para las sesiones en línea y las herra- 
mientas como Mapa de Empatía, Escenarios de Usos, Presentación de exposiciones para inversores conocida como Pithc a través del video, los cuales constituyeron en aspectos clave para articular el B-Learning y el Modelo PACIR (Presencia, Alcance, Capacitación, Interacción y E-Learning) en la formación de competencias en investigación aplicadas e innovación en los estudiantes de Tecnología en electrónica de la Universidad de Caldas objeto de estudio.

También comprendió la recolección de información al inicio mediante la administración de la pre prueba a las cuatro secciones y al concluir la aplicación del programa con la pos prueba a las mismas secciones, para conocer si el programa mejoró el logro de aprendizaje en las competencias en investigación aplicada e innovación para los estudiantes.

\section{Confidencialidad}

Para garantizar la información proporcionada por la muestra, primero se solicitó el consentimiento a la Dirección de la Universidad, la misma que se encargó de solicitar la autorización respectiva a las personas a cargo de los menores. Así mismo, se garantizó la protección de la identidad de la muestra de estudio, en respeto a sus derechos individuales.

\section{Resultados}

En el análisis de los resultados se describe en un primer momento los niveles de aprendizaje de las competencias en investigación aplicada e innovación, de forma general; luego se hace la comparación entre los grupos de control y experimental. A continuación, se presentan los siguientes hallazgos.

Aprendizaje de las competencias en investigación aplicada e innovación

Según la tabla 5, en el pre test la mayoría de estudiantes del grupo de control (80\%) y del grupo experimental (60\%) se encontraban en inicio. En el post test, la mayoría de los estudiantes del grupo de control $(60 \%)$ se encontraban en proceso, mientras que en el grupo experimental el $40 \%$ alcanzó el logro destacado y el 30\% el logro previsto.

Tabla 5

Aprendizaje de las Competencias en Investigación Aplicada e Innovación según niveles de logro, pre test y post test

\begin{tabular}{lllllr}
\hline \multirow{2}{*}{ Fase } & \multicolumn{2}{l}{$\begin{array}{l}\text { Nivel } \\
\text { de } \\
\text { Apren- } \\
\text { dizaje }\end{array}$} & \multicolumn{1}{l}{ Grupo de Control } & Grupo Experimental \\
\hline Pre & Alto & Destacado & $3 \%(1)$ & Destacado & $5 \%(2)$ \\
\cline { 2 - 6 } test & Medio & Previsto & $7 \%(3)$ & Previsto & $10 \%(4)$ \\
\cline { 2 - 6 } & Bajo & En proceso & $10 \%(4)$ & En proceso & $15 \%(6)$ \\
\cline { 2 - 6 } Post & Nulo & Inicio & $80 \%(29)$ & Inicio & $60 \%(27)$ \\
\cline { 2 - 6 } test & Mlto & Destacado & $6 \%(2)$ & Destacado & $40 \%(16)$ \\
\cline { 2 - 6 } & Medio & Previsto & $14 \%(6)$ & Previsto & $30 \%(11)$ \\
\cline { 2 - 6 } & Bujo & En proceso & $60 \%(21)$ & En proceso & $20 \%(8)$ \\
\hline
\end{tabular}

Fuente: Base de Datos de los resultados listas de chequeo según entregables del proceso en Campus Virtual Universidad de Caldas.

Nivel de logro en el aprendizaje de las competencias en investigación aplicada e innovación por secciones

De acuerdo con los resultados que se muestran en la tabla 6, en el nivel Nulo (Inicio), los mayores porcentajes de estudiantes estuvo en 4 Semestre de Electrónica 2 Riosucio (19.7\%) y 5 Semestre Electiva 1 Dorada (15.8\%); en el nivel Bajo (En proceso), los mayores porcentajes de mujeres corresponden a los grupos de 6 Semestre de Electiva 3 Dorada (6.6\%) y Manizales (5.3\%). El Nivel Medio (Previsto) tuvo mayor representación de logro en el 6 Semestre de Electiva 3 de Manizales (9.2\%); en el nivel Alto (Destacado), el mayor porcentaje de estudiantes se registró en el $6 \mathrm{Se}$ mestre de Electiva 3 de Manizales (10.5\%) seguido de la Dorada (7.9\%).

La comparación gráfica de los resultados observada en la figura 1, muestra que en tercer grado A es muy evidente que los estudiantes de $4 \mathrm{Se}$ mestre de Electrónica 2 de Riosucio se hallan en inicio $(19.7 \%)$ para desarrollar sus competencias en investigación aplicada e innovación; en el 5 Semestres de Electiva 1 de la Dorada, un porcentaje acumulado de $22.4 \%$ (la mayoría) están entre nivel nulo (inicio) y nivel bajo (en proceso); en 6 
Tecnologías de Información y comunicación desde la virtualidad para la formación en investigación aplicada e innovación "caso semilleros de investigación en los programas tecnológicos Universidad de Caldas"

Tabla 6

Nivel de Logro Aprendizaje de las Competencias en Investigación Aplicada e Innovación por secciones

\begin{tabular}{|c|c|c|c|}
\hline Fase & Niveles de Logro & $\mathrm{F}$ & $\%$ \\
\hline \multirow{2}{*}{$\begin{array}{l}5 \text { Electiva } \\
1 \text { Dorada }\end{array}$} & Alto (Destacado) & 2 & $2,6 \%$ \\
\hline & Medio (Previsto) & 3 & $3,9 \%$ \\
\hline \multirow[t]{2}{*}{$N=22$} & Bajo (En Proceso) & 5 & $6,6 \%$ \\
\hline & Nulo (Inicio) & 12 & $15,8 \%$ \\
\hline \multirow{2}{*}{$\begin{array}{l}6 \text { Electiva } \\
3 \text { Dorada }\end{array}$} & Alto (Destacado) & 6 & $7,9 \%$ \\
\hline & Medio (Previsto) & 4 & $5,3 \%$ \\
\hline \multirow[t]{2}{*}{$N=16$} & Bajo (En Proceso) & 4 & $5,3 \%$ \\
\hline & Nulo (Inicio) & 2 & $2,6 \%$ \\
\hline \multirow{3}{*}{$\begin{array}{l}6 \text { Electiva } \\
3 \text { Maniza- } \\
\text { les }\end{array}$} & Alto (Destacado) & 8 & $10,5 \%$ \\
\hline & Medio (Previsto) & 7 & $9,2 \%$ \\
\hline & Bajo (En Proceso) & 4 & $5,3 \%$ \\
\hline $\mathrm{N}=21$ & Nulo (Inicio) & 2 & $2,6 \%$ \\
\hline \multirow{3}{*}{$\begin{array}{l}4 \text { Elec- } \\
\text { trónica } 2 \\
\text { Riosucio }\end{array}$} & Alto (Destacado) & 0 & $0,0 \%$ \\
\hline & Medio (Previsto) & 1 & $1,3 \%$ \\
\hline & Bajo (En Proceso) & 1 & $1,3 \%$ \\
\hline \multirow[t]{2}{*}{$N=17$} & Nulo (Inicio) & 15 & $19,7 \%$ \\
\hline & Total & 76 & $100 \%$ \\
\hline
\end{tabular}

Fuente: Base de Datos de los resultados listas de chequeo según entregables del proceso en Campus Virtual Universidad de Caldas.

Semestre de Electiva 3 Manizales un promedio acumulado del $19.7 \%$ están en nivel Alto (Destacado) y Medio (Previsto) y en 6 Semestre de Electiva 3 Dorada, un porcentaje acumulado de $13.2 \%$ están entre los niveles Alto (Destacado) y Medio (Previsto).

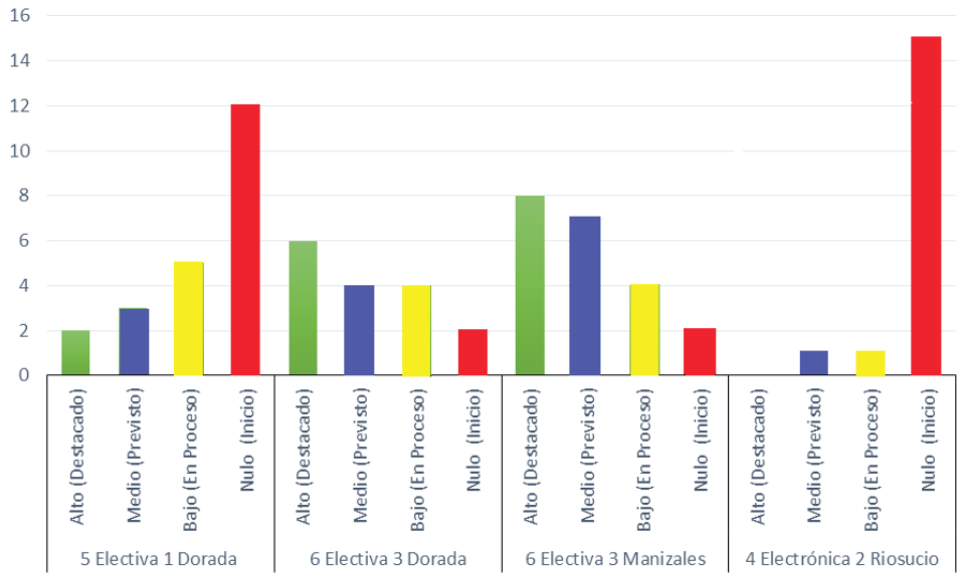

Figura 1. Comparación nivel de Logro Aprendizaje de las Competencias en Investigación Aplicada e Innovación por secciones.
Los resultados del proyecto se validaron por el Grupo de Investigación ReNuevaTe Ciencia Tecnología e innovación categorizado en $\mathrm{C}$ y Reconocido por COLCIENCIAS, su fiabilidad se demostró mediante la presentación de 35 proyectos por parte de los estudiantes donde presentaron sus prototipos funcionales y exposición de póster por proyecto en 2 eventos promovidos por la Universidad de Caldas y financiado por la Facultad de Ciencias Exactas y Naturales, Programa Tecnología en Electrónica en el Municipio de La Dorada el 4 de Mayo de 2017 y Municipio de Manizales 1 de Junio de 2017 denominado la Universidad Investiga - Investigación, Desarrollo e Innovación, cuyo propósito es realizar divulgación de las capacidades de la Universidad de Caldas y demás instituciones o investigadores convocados en el área de investigación, desarrollo tecnológico e innovación, mediante ponencias, talleres, concurso de robótica y exposición de proyectos tecnológicos.

\section{Discusión y Conclusiones}

Las universidades como fuentes de conocimiento requieren incorporar en sus planes de gestión la transferencia de conocimiento, para acercarse comprensivamente a los clientes naturales consumidores de información y conocimiento. Por lo que las universidades deben considerar que en los programas tecnológicos el incluir realizar investigación aplicada, lo cual permita seguir estimulando la creación y desarrollo de semilleros de investigación, para dimensionar la capacidad de desarrollos tecnológicos y producción científica que se pueden realizar desde estos programas contribuyendo al desarrollo integral de los estudiantes y la comunidad en general. Junto a ello también es relevante referir que el implementar nuevas metodologías y estrategias didácticas, acercando a los estudiantes a la formación en investigación a través de los semilleros promueva la innovación en los estudiantes, como se ha podido contrastar en este estudio al encontrar diferencias entre el grupo control y experimental después de la aplicación de la experimentación al haber obtenido el $40 \%$ de la muestra del grupo experimen- 
tal el nivel de logro destacado, 30\% en logro previsto. Estos resultados nos llevan a la reflexión de las mejoras que se puede obtener en implementar nuevas metodologías y estrategias en el aula a través de los semilleros de investigación.

Se observa que hay resultados diferenciados entre el grupo de control y experimental, al haber estudiantes que estuvieron entre el logro previsto y el logro destacado del grupo experimental, con lo que se puede incidir que las tecnologías de la información y comunicación unidas a la investigación a través de los semilleros cumplen un rol importante en el desarrollo de competencias de los estudiantes, como también lo asevera López \& Toro (2017) al enfatizar que los semilleros contribuyen a alcanzar los objetivos de la educación superior y sirven de estrategia para la iniciación en la actividad científica.

Igualmente, en concordancia con el estudio de Rojas, Silva \& Correa (2014) sobre tecnologías de la información y la comunicación en la educación encontramos que las TIC mejoran las formas de enseñar y el aprendizaje de los estudiantes a través de la apropiación de las tecnologías dentro de las cuales está el blogs, internet, etc., potenciando los procesos de enseńanza-aprendizaje, y si esto va unido a los semilleros de investigación según Alvites-Huamani (2015) crea espacios de análisis, reflexión y aprendizaje de una cultura investigativa y que los estudiantes pongan en práctica competencias y actividades investigativas.

Este estudio al utilizar las tecnología permitió cimentar la línea de Investigación "Educación, Ingeniería y Tecnologías de Información y Comunicación" para el desarrollo de proyectos en las áreas de especialidad de los programas Tecnológicos de la Universidad de Caldas y se gestionó la vinculación de los semilleros de investigación al Grupo de Investigación: ReNuevaTe Ciencia, Tecnología e Innovación, (Grupo Externo), como la implementación del Semillero de Investigación e Innovación TECSIS y RELEC como eje articulador desde la virtualidad, dentro de los cuales se desarrolló recursos desde las TIC articulados con herramientas como Blackboard Collaborate, Whatsapp, Neolms, Moodle, Blogger, Soundcloud, Youtube, Vimeo entre otros, que se ha podido contrastar al haber obtenido que los niveles de logro se incrementan porcentualmente al aplicar las TIC a través de los semilleros de investigación, lo que conlleva a servir como medios en la mejora del proceso enseñanza-aprendizaje, resultados que se relacionan con lo investigado por Farrońay \& Díaz (2016) al concluir que las TIC son herramientas que ayudan a mejorar el proceso educativo y la gestión del conocimiento.

Para investigaciones futuras sería importante hacer estudios longitudinales para hacer el seguimiento de los estudiantes que se fueron formando en el semillero de investigación y conocer si continuaron en grupos de investigación o formaron ellos sus propios grupos y que resultados obtuvieron.

\section{Agradecimiento}

Los avances e iniciativa para el desarrollo de este proyecto en los programas Tecnológicos de la Universidad de Caldas, su estrategia e impulso se debe al Ingeniero Fabio Andrés López Salazar Coordinador de la Tecnología en Sistemas Informáticos, con quien el autor ha trabajado desde el año 2016 y que ha permitido alcanzar avances importantes para las Tecnologías que se consideraban para la comunidad académica que no podían investigar ante sus limitaciones de Tiempo, Espacio y Recursos.

\section{REFERENCIAS BIBLIOGRÁFICAS}

Aguerrondo, I. (2017). El nuevo paradigma de la educación para el siglo XXI.

Alvites-Huamaní, C. (2015). Creación e implementación de semilleros de investigación en la dirección universitaria de educación a distancia de la Universidad Alas Peruanas. Revista Hamut'ay, 2(1), 63-70. https://doi.org/10.21503/ hamu.v2i 1.852

Aprende en Línea (8 abril de 2018) Las TIC como apoyo a la educación. Recuperado de http://aprendeenlinea.udea. edu.co $/ \mathrm{lms} /$ investigacion $/ \mathrm{mod} / \mathrm{page} /$ view.php?id=3118

Cauas, D. (2015). Definición de las variables, enfoque y tipo de investigación. Bogotá: biblioteca electrónica de la universidad Nacional de Colombia.

Llamas, J. (2007). Importancia de los semilleros de investigación en la universidad de Cartagena. Revista Palobra,"palabra que obra”, 7(7), 137-141. Recuperado de http:// 
Tecnologías de Información y comunicación desde la virtualidad para la formación en investigación aplicada e innovación "caso semilleros de investigación en los programas tecnológicos Universidad de Caldas"

revistas.unicartagena.edu.co/index.php/palobra/article/ view/159/124

Carrasco, Y. (2017). Gestión de la innovación y el desarrollo tecnológico en la gran minería a tajo abierto del Perú: estudio de casos. (Tesis de Maestría). Pontificia Universidad Católica del Perú. Recuperado de http://tesis.pucp.edu.pe/repositorio/bitstream/handle/123456789/8521/CARRASCO_ MERMA_GESTION\%20de\%20la_INNOVACION\%20 y\%20el\%20desarrollo.pdf?sequence $=1$ \&isAllowed $=y$

Chávez, J., Del Toro, M. \& López, O. (2017) Blog, correo electrónico y foros temáticos: $\mathrm{Su}$ uso, dominio y actitud en estudiantes de educación medio superior de México. Hamut'ay, 4 (2), 45-54. https://doi.org/10.21503/hamu. v4i2.1471

De Oslo, M. (2016). Guía para la recogida e interpretación de datos sobre innovación. Organización de Cooperation y Desarrollo Económico. (OCDE). Oficina de Estadística de las Comunidades Europeas. Recuperado de http://www.itq. edu.mx/convocatorias/manualdeoslo.pdf

Farroñay, P. \& Ancaya, M. (2016) Gestión administrativa y conocimiento de las TIC en docentes de educación primaria de las instituciones educativas Innova Schools de San Juan de Lurigancho y Ate. Revista Hamut'ay, 3 (1), 31-45. https://doi.org/10.21503/hamu.v3i1.998

Grande, M., Cañon, R. \& Cantón, I. (2016) Tecnologías de la información y la comunicación: evolución del concepto y características. Internacional Journal of Education Research and Innovation, 6, 218-230. Recuperado de https:/www. upo.es/revistas/index.php/IJERI/article/view/1703/1559.

Instituto Tecnológico y de Estudios Superiores de Monterrey (2010) Características de una técnica didáctica. Centro virtual de técnicas didácticas. Recuperado de http://sitios. itesm.mx/va/dide2/tecnicas_didacticas/caract_td.htm

Izquierdo, B., Juárez, G. \& Salas, B. (2017). Importancia de las tecnologías en los trabajos de investigación: Una experiencia en los estudiantes de Licenciatura, Área económico-administrativa de la Universidad Veracruzana-México. Hamut'ay, 4 (1), 9-17. https://doi.org/10.21503/hamu. v4i1.1392

Lazarsfeld, P. (1973). De los Conceptos a los Índices Empíricos en Boudon R. \& Lazarsfeld P., Metodología de las Ciencias Sociales, pp. 36-46. Editorial Laia, Barcelona.

Larreal, B. (2015). Herramientas de comunicación para el desarrollo de la inteligencia lógica matemática. Revista Opción, número especial 3, 715-734. Recuperado de http:// www.redalyc.org/html/310/31045567037/.

López, Y. \& Toro, N. (2017) El semillero de investigación, una alternativa innovadora en el sistema educativo colombiano. Revista Universitaria Ruta, 19 (2), 31-47. Recuperado de http://revistas.userena.cl/index.php/ruta/article/ view/914/pdf_8

Lozada, J. (2014). Investigación aplicada: Definición, propiedad intelectual e industria. CienciAmérica: Revista de divulgación científica de la Universidad Tecnológica Indoamérica, 3(1), 47-50. Recuperada de http://www.cienciamerica. us/openjournal/index.php/uti/article/view/30/23

Marín-Díaz, V. \& Gómez-Parra, E. (2015). Edublogs in the development of the European higher education area: an initiative in the Primary Education Degree course at the University of Cordoba (Spain). 7th World Conference on Educational Sciences, (WCES-2015), 05-07, February 2015, Novotel Athens Convention Center, Athens, Greece. https://doi.org/10.1016/j.sbspro.2015.07.329

Gualsaqui, L. (2015). Las estrategias didácticas apoyadas en las tecnologías de la información y comunicación y el proceso de enseńanza-aprendizaje de la asignatura de informática en los primeros cursos de bachillerato general unificado del régimen costa del Liceo Naval de Quito, (Tesis de Maestría) Universidad Técnica de Ambato, Ecuador. Recuperado de http://repositorio.uta.edu.ec/jspui/bitstream/123456789/17255/1/LEGM\%20-\%20TESIS\%20 LICQUI\%20-\%20ABR2015.pdf

Morales, R. \& Pereida, M. (2017). Inclusión de estilos de aprendizaje como estrategia didáctica aplicada en un AVA. Campus Virtuales, 6(1), 67-75. Recuperado de http:// uajournals.com/ojs/index.php/campusvirtuales/article/ view/188/161

Padilla, J., Rincón, D., \& Buitrago, L. (2015) La investigación formativa desde la teoría de las representaciones sociales en la Facultad de Estudios a Distancia de la Universidad Militar Nueva Granada. Recuperado de Revista Academia y Virtualidad, 8 (1), 21-34. https://revistas.unimilitar.edu.co/ index.php/ravi/article/view/443/239

Porras, I. (2017). Redes Sociales, Facebook y Blog según los Estilos de Aprendizaje en Cursos E-Learning. Hamut'ay, 4 (1), 60-74. https://doi.org/10.21503/hamu.v4i1.1395

Ramírez, J., \& Vega, O. (2015). Las TIC como factor vinculante innovador de desarrollo nacional y organizacional en Venezuela. COEPTUM, 7(2), 94-111.

Riaño, C. (2017). Diseño de un Modelo de Transferencia del Conocimiento para el Manejo del Sector Autopartista en Bogotá. (Tesis de Maestría) Universidad Distrital Francisco José de Caldas, Colombia. Recuperado de http://repository.udistrital.edu.co/bitstream/11349/7238/1/TESIS\%20 CLAUDIA\%20RIA\%C3\%91O\%20VERSION\%20TERMINADA\%2015\%208.pdf

Rivera, J. (2011) Impacto de las tecnologías de información y comunicación en los procesos de enseñanza-aprendizaje. Investigación Educativa, 15 (27), 127-137. Recuperado de http://sisbib.unmsm.edu.pe/bibvirtual/publicaciones/ inv_educativa/2011_n27/a08v15n27.pdf

Rojas, M. (2017). Los recursos tecnológicos como soporte para la enseńanza de las ciencias naturales. Hamut'ay, 4 (1), 85-95. https://doi.org/10.21503/hamu.v4i1.1403

Rojas, M., Silva, A. \& Correa, L. (2014) Tecnología de la información y la comunicación en la educación: Tendencias investigativas. Revista Academia y Virtualidad 7 (2), 27-40. Recuperado de https://revistas.unimilitar.edu.co/index.php/ ravi/article/view/316/199

Salas-Arbeláez, L., Solarte, M. \& Vargas, G. (2017). Efecto 
de la cultura organizacional en el rendimiento de las PYMES de Cali. Suma de Negocios, 8(18), 88-95. https://doi.org/10.1016/j.sumneg.2017.11.006

Ruiz, N., Mendoza, M. \& Ferrer, L. (2014) Influencia de las tecnologías de la información y comunicación en los roles e interrelaciones entre estudiantes y docentes en programas presenciales de educación superior. Hallazgos, 11 (22), 435-454. Recuperado de http://www.scielo.org.co/pdf/hall/ v1 $\ln 22 / \mathrm{v} 11 \mathrm{n} 22 \mathrm{a} 23 . \mathrm{pdf}$

Suelves, D. (2017). Pedagogía Red. Una educación para tiempos de internet. Revista Iberoamericana de Educación, 74(1), 10. Recuperado de https://rieoei.org/RIE/article/ view/634

UEB-Universidad Estatal de Bolívar (2013) Reglamento de Semilleros de Investigación de la Universidad Estatal de Bolívar. Recuperado de http:/www.ueb.edu.ec/sitio/images/ PDF/REGLAMENTOS/2013/REGLAMENTO_DE_ SEMILLEROS_DE_INVESTIGACION.pdf

Villalba, J. \& Gonzáles, A. (2017) La importancia de los semilleros de investigación. Revista Prolegómenos, 20 (39), 9-10. Recuperado de http://www.scielo.org.co/pdf/prole/ v20n39/v20n39a01.pdf 